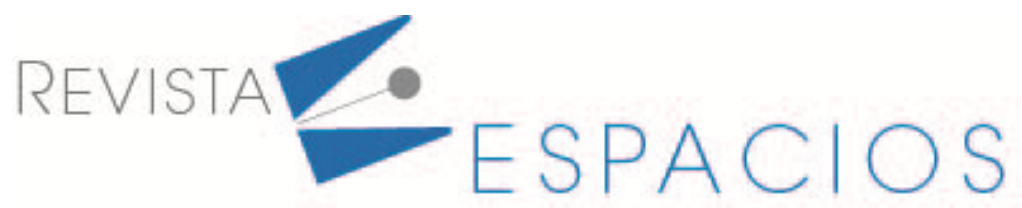

\title{
Estimación de la demanda de energía eléctrica de la ciudad de Ibagué, Colombia, por medio de un modelo ARDL
}

\section{Estimation of the electricity demand of the city of Ibagué-Colombia, by means of an ARDL model}

ALZATE, Juan C. ${ }^{1}$

DÍAZ, Roberto A. ${ }^{2}$

BENAVIDES, Oscar A. ${ }^{3}$

VERA, José A. ${ }^{4}$

\section{Resumen}

El trabajo de investigación estima la demanda por energía eléctrica en la ciudad de lbagué para los diferentes agentes utilizando datos trimestrales en el periodo 2005-2019, para lo cual elabora modelos autorregresivos de retardos distribuidos -ARDL. Los cuales permiten hacer estimaciones pertinentes para el corto y largo plazo. Se obtuvieron las ecuaciones de las demandas, donde se identifica que la mayoría de agentes presenta significancia referente al precio de la energía y de la tasa de ocupación.

Palabras Claves: demanda de energía; agentes y modelos.

\begin{abstract}
The research work estimates the demand for electricity in the city of Ibague for the different agents using quarterly data in the period 2005-2019, for which it develops autoregressive models of distributed delays -ARDL. Which allow making pertinent estimates for the short and long term. The demand equations were obtained, where it is identified that the majority of agents present significance regarding the price of energy and the occupancy rate.

Keywords: energy demand; agents and models.
\end{abstract}

\section{Introducción}

El presente trabajo de investigación estima la demanda por energía eléctrica en la ciudad de Ibagué para los diferentes agentes demandantes utilizando datos trimestrales en el periodo 2005-2019, para lo cual elabora modelos autorregresivos de retardos distribuidos -ARDL. La pretensión es que los modelos propuesto permitan hacer estimaciones pertinentes para el corto y largo plazo. Ahora bien, para identificar y plantear el modelo econométrico es preciso definir antes un modelo teórico, que relacione las variables relevantes en la determinación de la demanda de energía eléctrica; las elasticidades en precio de la demanda, así como los efectos que sobre el crecimiento económico tiene la dinámica del subsector de la energía eléctrica y la

\footnotetext{
${ }^{1}$ Empresarios. Consultor. Privado. juancarlosalzateosorio@gmail.com

2 Docente. ECACEN. Universidad Nacional Abierta y a Distancia-UNAD. roberto.diaz@unad.edu.co

${ }^{3}$ Docente. ECACEN. Universidad Nacional Abierta y a Distancia-UNAD. oscara.benavides@unad.edu.co

${ }^{4}$ Docente. ECACEN. Universidad Nacional Abierta y a Distancia-UNAD. jose.vera@unad.edu.co
} 
comprensión general de la estructura de mercado (regulaciones, discriminación de precios) permite entender mejor la demanda de este servicio público domiciliario.

El objetivo principal de la investigación es la identificación de los determinantes de la variación en el consumo de energía eléctrica para la ciudad de lbagué, y los objetivos específicos estipulados son: establecer las variables necesarias y la proyección para el crecimiento de la demanda de energía eléctrica en dicha ciudad; construir y verificar modelos econométricos de la demanda de energía eléctrica, basada en datos trimestrales de los años 2005 al 2019, y por último la determinación de las variables significativas que sirvan de insumo para la implementación de un modelo de proyección de demanda de energía eléctrica.

Como primera medida se recopiló el cuerpo teórico sobre la relación entre demanda por energía eléctrica y crecimiento económico. La evolución material de la humanidad ha estado marcada por la utilización de manera creciente y cada vez más eficiente de energía obtenida a partir de recursos naturales, lo cual permitió desarrollar grandes avances como las revoluciones agrícola e industrial y en ese sentido aumentar cada vez más la capacidad productiva, brindando la oportunidad a las sociedades de crecer a tasas aceleradas, pero con el tiempo ha traídos grandes repercusiones, como el aumento del efecto invernadero y polución en las grandes ciudades, donde se producen una serie de externalidades.

El marco teórico del presente trabajo desarrolla la relación de los precios en la demanda de energía para mercados, especialmente mercados regulados, con los cual se construye un referente analítico para el mercado energético en la ciudad de Ibagué, seguidamente se analizan los datos suministrados por la Superintendencia de Servicios Públicos, por metodologías cuantitativas.

Las técnicas econométricas utilizadas en este trabajo investigativo son los modelos Autorregresivos de Retardos Distribuidos -ARDL, mediante la utilización del software de análisis de datos Eviews, de igual manera se estimaron elasticidades a corto y largo plazo.

\section{Metodología}

El diseño metodológico permite reconocer de forma detallada los pasos sistemáticos y rigurosos para el logro de los objetivos planteados; abarcando la organización de los datos de series de tiempo hasta la determinación del mejor modelo para el tratamiento de la información de manera fiable y rigurosa.

La información está estructurada con los datos trimestrales de las variables del consumo de energía y las variables relacionadas a los determinantes de la demanda de energía eléctrica, las cuales fueron extraídas del Sistema Único de Información de la Superintendencia de Servicios Públicos Domiciliarios -SUI- y para el caso de la Tasa de Ocupación se descargó del Departamento Administrativo Nacional de Estadística -DANE, con registros desde el año 2005 a 2019.

Para la modelación econométrica por series de tiempo y específicamente por medio de los modelos de autorregresivos de retardos distribuidos ARDL se emplean una serie de datos con una periodicidad trimestral desde el año de 2005 hasta el año de 2019. De igual manera para efectos del crecimiento económico en la ciudad de Ibagué y debido a que los datos de las cuentas regionales solo hay datos del Producto Interno Bruto de forma anualizada se tuvo que emplear la Tasa de Ocupación del Empleo para la ciudad de Ibagué calculada para cada trimestre. las variables utilizada en el estudio son el consumo promedio trimestral en Kgh del sector comercial Inconcomer; consumo promedio trimestral del sector industrial -Inconindustrial; consumo promedio trimestral del sector oficial-Inconoficial; el consumo promedio trimestral del sector residencial-Inconresidencial y la tasa de ocupación en la ciudad de Ibagué - Into y las variables del precio de cada sector analizado (Intareidencial, Intacomercial, Intaindustrial y Intaoficial las cuales fueron deflactadas con el Índice de los Precios al Productor), a las variables se les realizará una serie de transformaciones para reducir la varianza y la tendencia, seguidamente 
se práctica una prueba de raíz unitaria para determinar si las variables son o no estacionarias, lo cual permite tener indicios sobre el proceso generador de las series y el posible método de tratamiento de los datos.

Se realizaron los tests de raíz unitaria en cada una de las variables aplicando el test del Argumento de DickeyFuller -ADF con tendencia e intercepto, fue aplicado a las variables en niveles y diferenciadas. Las variables se les realizó una transformación por logaritmo neperiano, lo cual en términos económicos al ser aplicado a una serie económica se puede lograr reducir la heterosedasticidad, reducir la distancia entre valores extremos y se podría mejorar la potencia de los test estadísticos. Los resultados de las pruebas de raíz unitaria sin tendencia en las variables arrojan que todas son integradas de orden (1), lo que es igual a decir que son estacionarias al aplicarles una primera diferencia.

Pesaran y Pesaran (1997); Pesaran y Smith (1998) y Pesaran et al. (2001) han introducido una técnica de cointegración alternativa conocida como la prueba Autoregressive Distributed Lag (ARDL). Esta técnica tiene un número de ventajas sobre las técnicas de cointegración de Johansen. Primero, el modelo ARDL es el enfoque más significativo estadísticamente para determinar la relación de cointegración en muestras pequeñas (Ghatak y Siddiki 2001), mientras que la cointegración de Johansen requiere grandes muestras de datos para su validez. Una segunda ventaja del enfoque ARDL es que, mientras que otras técnicas de cointegración requieren que todos los regresores estén integrados en el mismo orden; el ARDL se puede aplicar ya sea que los regresores sean I (1) y / o I (0).

\section{Resultados}

\subsection{Estado del arte}

Cardona y Ocampo (1989) adelantan una investigación para estimar la demanda de energía eléctrica del sector residencial en el área metropolitana de Medellín entre 1983 y 1987, bajo la coordinación del profesor Jesús Alonso Botero y con evidente influencia del trabajo de Taylor. Su gran particularidad, que es también su valor agregado, es que el análisis no es global (modelo de demanda nacional), sino local, donde evalúan varias herramientas econométricas y varias formas funcionales antes de realizar la estimación. Inicialmente realizan una teorización de la demanda de electricidad y se identifican las variables relevantes para el modelo. Refieren cómo el análisis es limitado dado que la información oficial utilizada presenta irregularidades en las series de tiempo.

Vélez, Botero y Yañez (1991) investigan las propiedades de la demanda residencial de energía eléctrica en dos ciudades colombianas (Bogotá y Medellín) en el periodo 1970-1983". Resaltan, como Taylor, Cardona y Ocampo que la modelación económica de este mercado tiene características especiales: discriminación de precios de los monopolios de distribución, lo que conlleva a que diferentes clases de consumidores enfrentan una curva de oferta ascendente que impide la agregación "horizontal" de la demanda y por ello se requiere de una agregación de las formas reducidas finales de las diferentes clases de consumidores. Al final de su ejercicio obtienen resultados econométricos estadísticamente significativos que guardan coherencia con los supuestos económicos convencionales.

Agostini, Plottier y Saavedra (2011) analizan la demanda de energía eléctrica para Chile, realizando una estimación en un contexto de una demanda creciente y una oferta aleatoria que genera riesgo de déficits inminentes. Su fin es identificar los determinantes de la demanda de energía eléctrica en los hogares chilenos, en particular las elasticidades precio e ingreso, analizando el grado se sustitubilidad entre energía eléctrica para uso residencial y gas licuado. La propuesta metodológica del trabajo es innovadora, aunque los resultados se afianzan en los presentados por trabajos previos y permiten inferir la conveniencia de una política de eficiencia energética en Chile, que logre mitigar los efectos negativos de los shocks de oferta. 
Para Argentina, Abril y Blanconá (2000) proponen un modelo parsimonioso para el comportamiento de la demanda diaria promedio de energía eléctrica que permita realizar pronósticos a corto plazo. El ejercicio de modelación asume un enfoque estructural de los modelos de espacio de estado. Se reconocen dificultades al modelar series de tiempo diarias, por ejemplo, en el ajuste del componente estacional, dado que se pueden presentar estacionalidades semanales y anuales. Para el caso de la estacionalidad semanal se elabora un modelo estructural, donde se involucran variables dummy. Cuando la estacionalidad es anual, se proponen modelos estructurales de series de tiempo porque los modelos tradicionales demandan un elevado número de parámetros, lo que impide el cumplimiento del principio de parsimonia. Se adelantan técnicas de spline.

Zapata, López y Rengifo (2008) consideran el efecto del modelamiento de la demanda en estudios de confiabilidad de largo plazo de sistemas eléctricos; estudio que utiliza registros de demanda de energía eléctrica de forma mensual por un espacio 29 años. Se construyen modelos de demanda populares en estudios de adecuación de SP para valorar su efecto sobre los índices de confiabilidad de dos sistemas de prueba y, sobre el tiempo computacional requerido en la técnica de simulación de Montecarlo secuencia. Utilizando registros mensuales de demanda se construyeron modelos para incorporarlos a la valoración de confiabilidad de dos sistemas de prueba.

Velásquez, Franco y Alonso (2009) proponen un modelo no lineal para la predicción de la demanda mensual de electricidad en Colombia, en un artículo del mismo nombre, que compara el desempeño de un modelo ARIMA, un perceptron multicapa y una red neuronal autorregresiva para pronosticar la demanda mensual de electricidad en Colombia, para el siguiente mes.

En un estudio más reciente de la UPME, titulado "Proyección de demanda de energía eléctrica en Colombia “(UPME, 2013) se ofrece una visión de la evolución histórica del consumo de energía eléctrica y potencia máxima en Colombia y de su prospectiva para las próximas dos décadas para realizar las proyecciones de demanda de energía eléctrica y potencia máxima.

Barreto y Ocampo en 2012 presentan un trabajo que estudió la relación a largo plazo entre el consumo de energía y el PIB en algunos países de América Latina, con control adicional por otros factores de producción como el capital y el trabajo (estimó una función de producción) para el período 1980 - 2009. Se empleó un modelo de datos panel no estacionario y cointegrado y logró concluir la existencia de una relación a largo plazo entre el PIB, el consumo de energía, el capital y el trabajo. Por su parte, los coeficientes estimados señalan que los países más sensibles a cambios en el factor de producción de energía en forma relativa son Panamá y Bolivia, en tanto que Colombia, Paraguay, Brasil y Ecuador manifiestan menor sensibilidad al uso, en términos relativos, de los otros factores.

\subsection{Características del consumo de energía eléctrica en la ciudad de lbagué}

En la siguiente gráfica se observa la evolución del consumo de energía eléctrica de la ciudad de lbagué agregado en dos sectores: total residencial y total no residencial. 
Figura 2

Tendencia de consumo de energía eléctrica en Kv en la ciudad de Ibagué residencial y no residencial. 2005-2019.

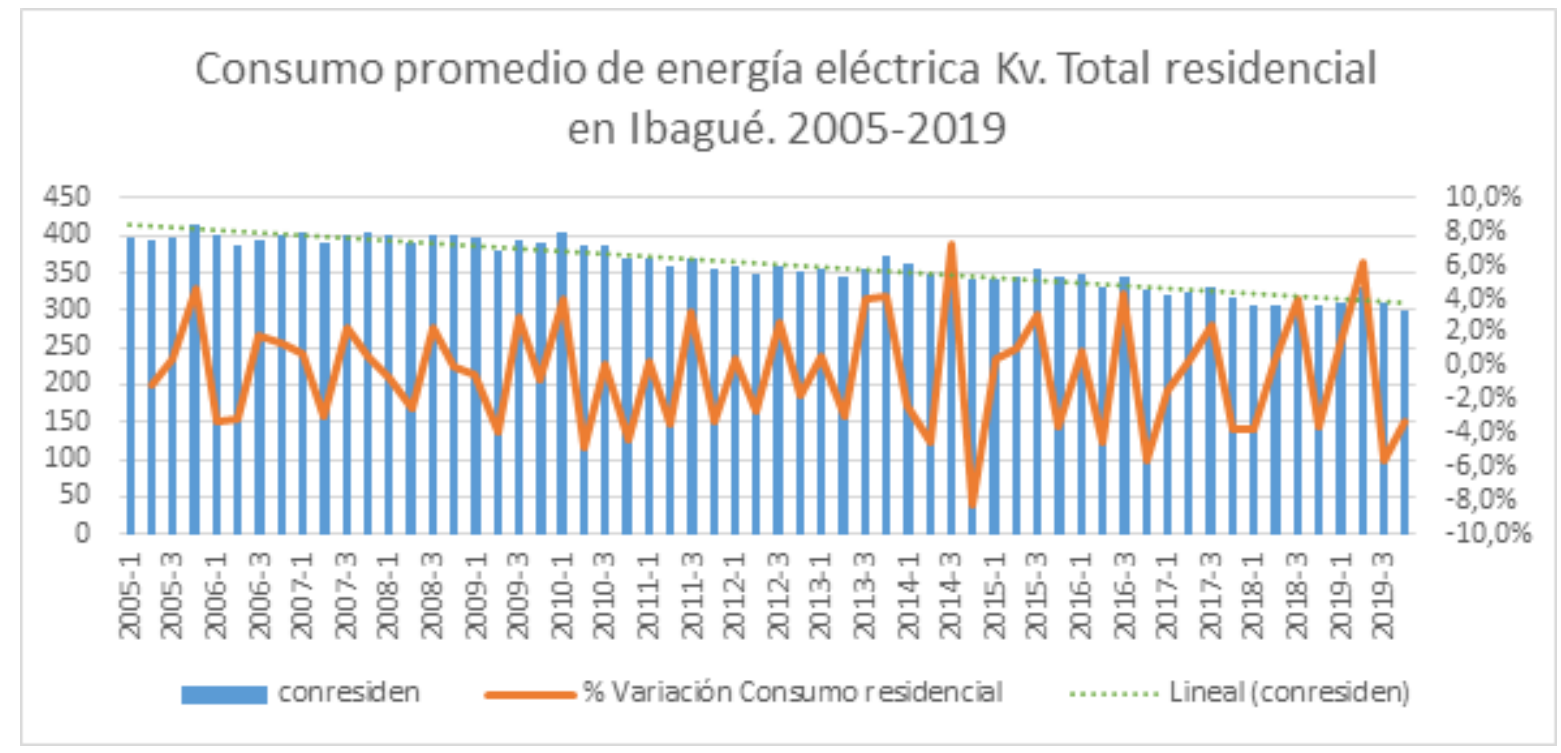

Fuente: Elaboración propia con base en los datos de la Superintendencia de Servicios Públicos Domiciliarios.

La figura No. 2, muestra una tendencia a disminuir el consumo de energía eléctrica en los clientes residenciales en la ciudad de Ibagué en el periodo de análisis. Con una fuerte caída en la tasa del consumo promedio para el cuarto trimestre del año 2014. En los primeros cinco años los residentes de la ciudad de Ibagué tienen un promedio de consumo trimestral de $395 \mathrm{Kvh}$, y del año 2015 a 2019 el promedio de consumo disminuye a 325,6 Kvh, para un reducción del 17,6\%, lo que denota que los hogares han implementado estrategias para reducir el consumo, como instalación de bombillas eficientes y la renovación de electrodomésticos con tecnologías de bajo consumo; al igual que la tarifa de la energía más alta induce a que las familias ibaguereñas tengan que consumir menos, y esto puede afectar la calidad de vida de dichas familias, especialmente los de estratos más bajos; lo cual amerita una recomendación de política económica para el país y la región, referente a mejorar la competitividad de todo el sistema eléctrico en el país, especialmente el precio a los diferentes consumidores, de cara de aumentar el bienestar y la competitividad de los demás agentes productivos, especialmente en la ciudad de Ibagué, que es una de las tres capitales del país que presenta una persistente tasa de desempleo.

En la siguiente figura se muestra la demanda de energía eléctrica para la ciudad de Ibagué clasificando por tipo de cliente. 
Figura 3

Demanda de energía eléctrica en la ciudad de Ibagué en Gvh, para clientes no residenciales. 2005-2019.

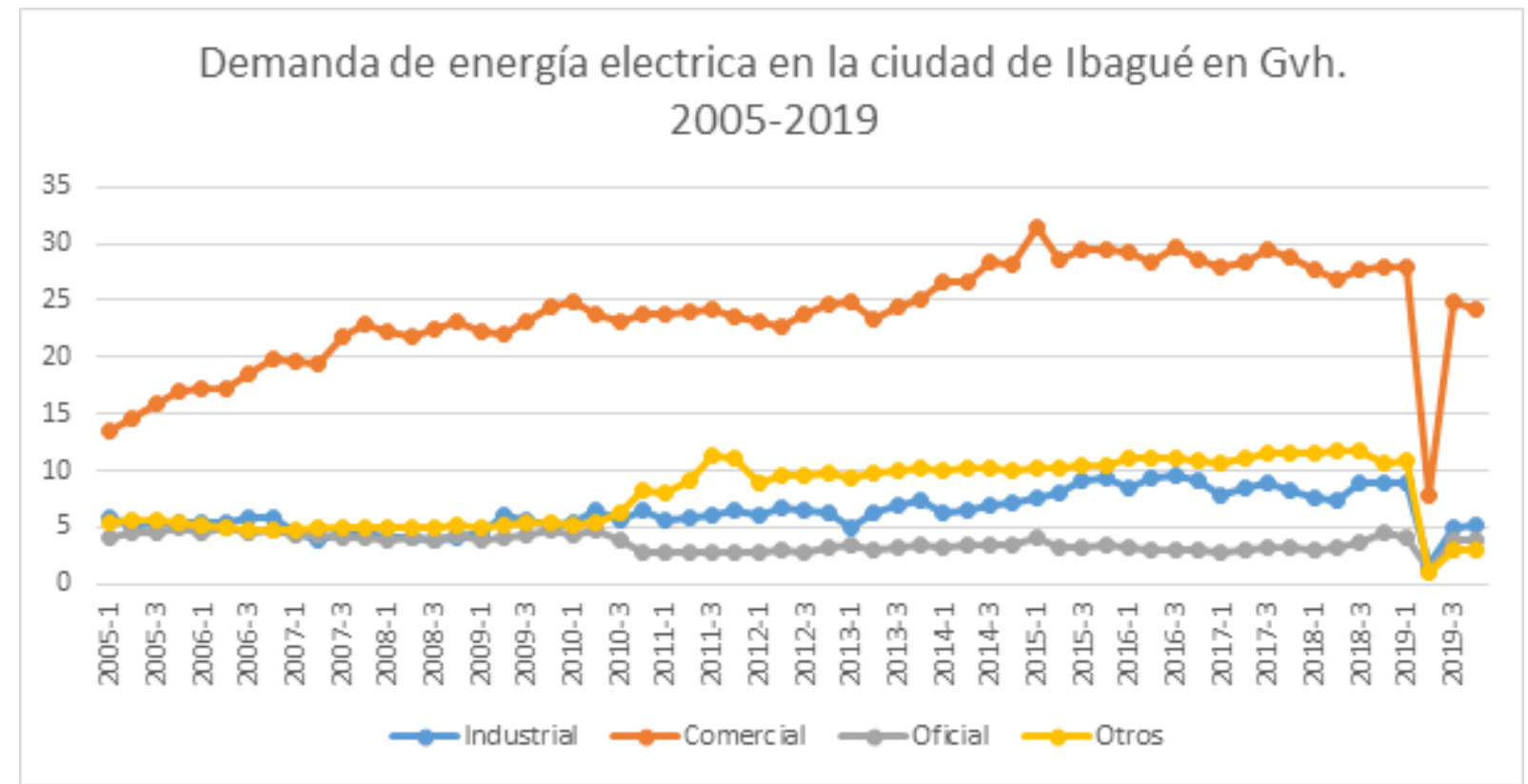

Fuente: Elaboración propia con base en los datos de la Superintendencia de Servicios Públicos Domiciliarios.

El mayor consumo durante el periodo de análisis del consumo y demanda de energía eléctrica en la ciudad de Ibagué corresponde de los clientes no residenciales, es el comercial, seguido de los clientes industriales y el menor consumo es el de los clientes institucionales u oficiales en la ciudad de lbagué. Se observa una tendencia en el incremento del consumo promedio de los sectores comerciales e industriales de la ciudad de lbagué en el periodo de análisis. Lo cual es un buen indicador de la actividad económica de la ciudad, especialmente de su mayor participación del sector comercial en el producto interno del municipio. Se presenta una fuerte caída en el consumo de energía eléctrica para el segundo trimestre del año de 2019, lo cual corresponde con la desaceleración que ha entrado la economía colombiana desde mediados de dicho año y que se afecta en mayor medida por la pandemia del Covid 19 en el año de 2020.

Se observa un crecimiento de las tarifas de la energía eléctrica en el periodo de análisis 2005-2019, en donde la residencial presenta un mayor valor, seguido de la comercial, la oficial y la tarifa del sector industrial es la de menor valor. Lo cual es preocupante para las familias y las empresas del sector industrial, que deberían de tener una menor tarifa de la energía eléctrica, para poder disminuir los costos de producción de los bienes y servicios y para que las familias tengan una mejor calidad de vida al gastar menos de la renta disponible en el pago del servicio de electricidad y alumbrado público en la ciudad de Ibagué. 
Figura 4

Tarifas de la energía eléctrica en la ciudad

de Ibagué en Kvh, por clientes. 2005-2019.

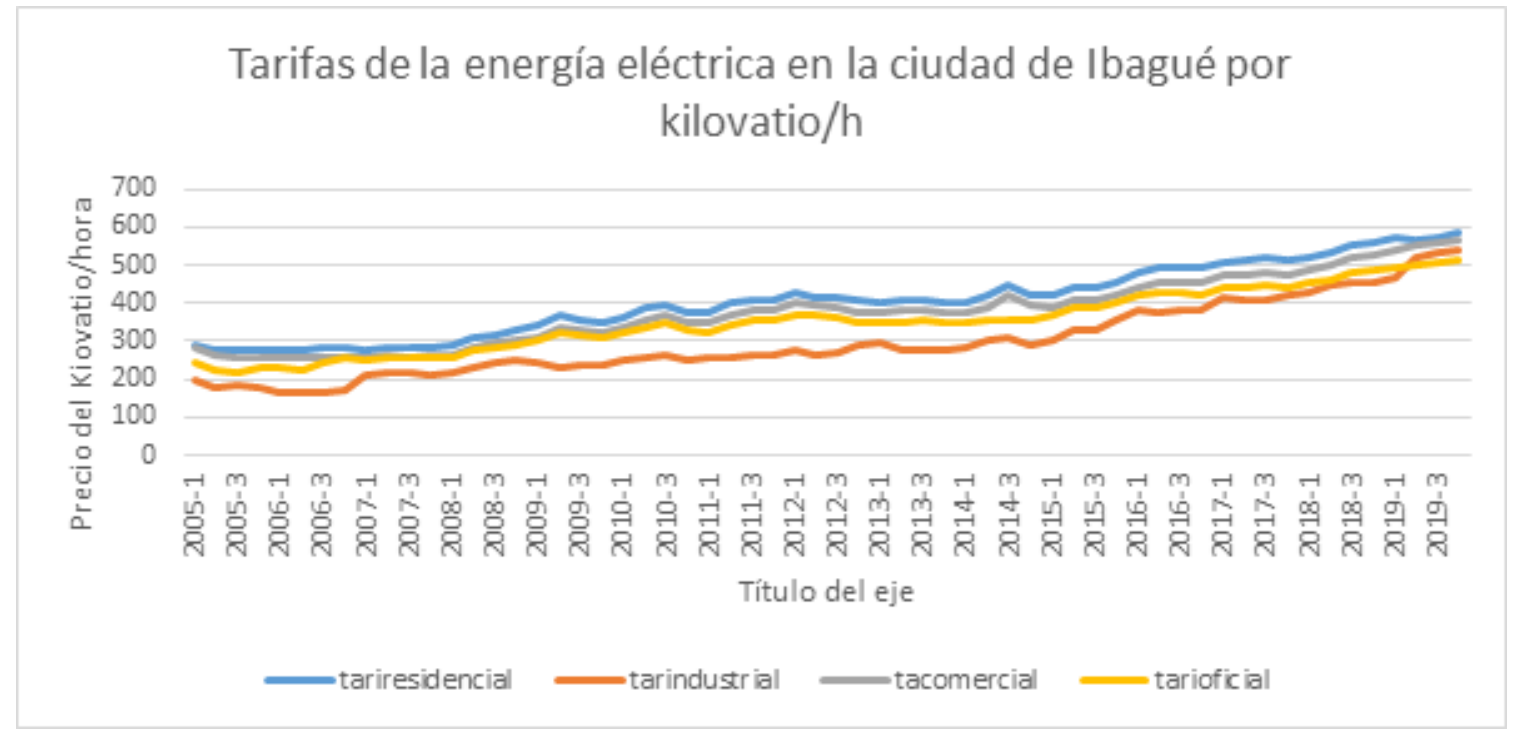

Fuente: Elaboración propia con base en los datos de

la Superintendencia de Servicios Públicos Domiciliarios

\subsection{Estructura general de los modelos ARDL}

Figura 5

Variables de consumo de energía del sector comercial, industrial, residencial y oficial, expresadas en logaritmos. 2005-2019.

Inconcomer

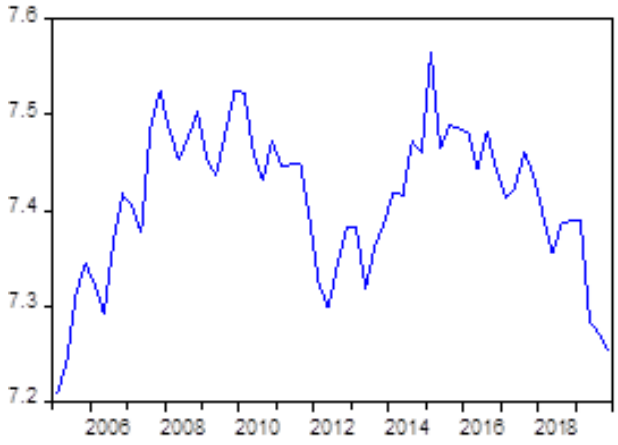

Inconoficial

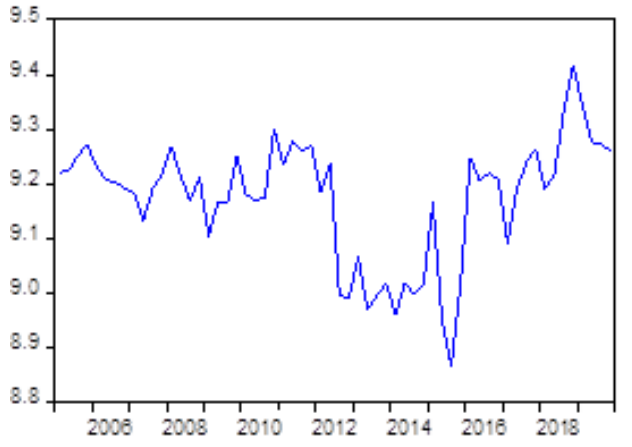

Inconindustrial

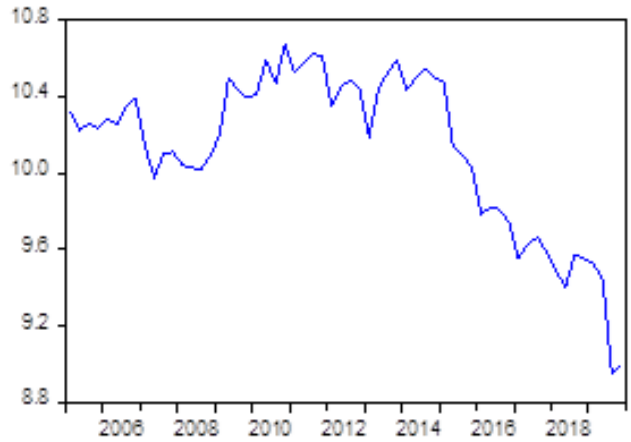

Inconresiden

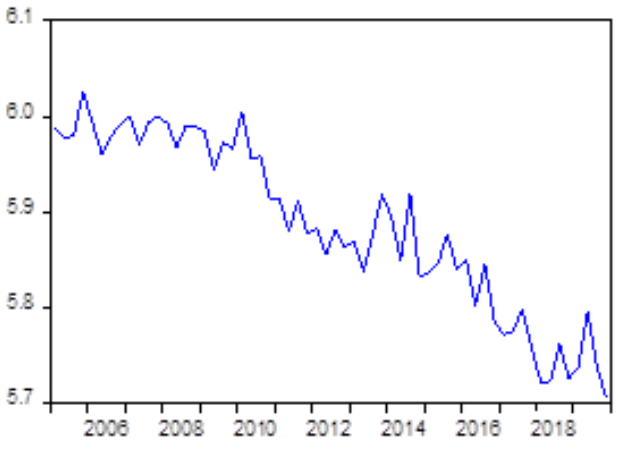


Figura 6

Variables de consumo de energía del sector comercial, industrial, residencial y oficial, expresadas en logaritmos y en primera diferencia. 2005-2019.

Differenced Incon comer
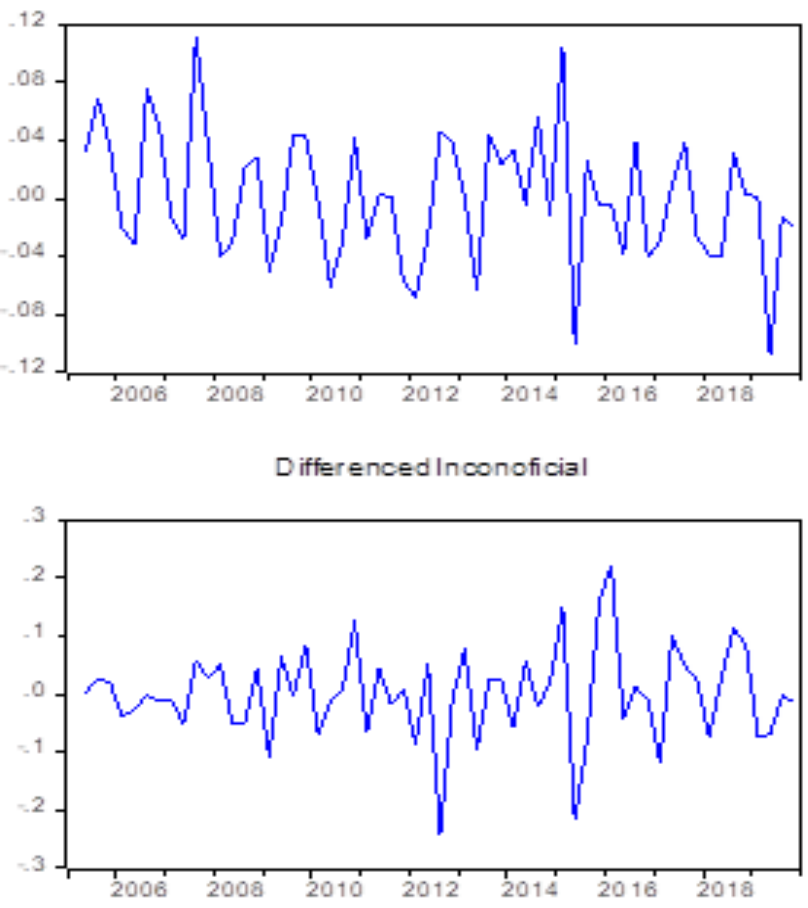

D ifferenced Inconindus trial

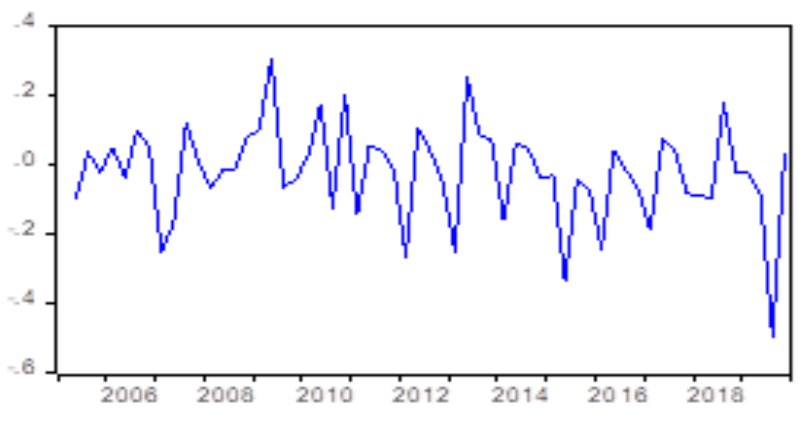

D ifferenced Inconres iden

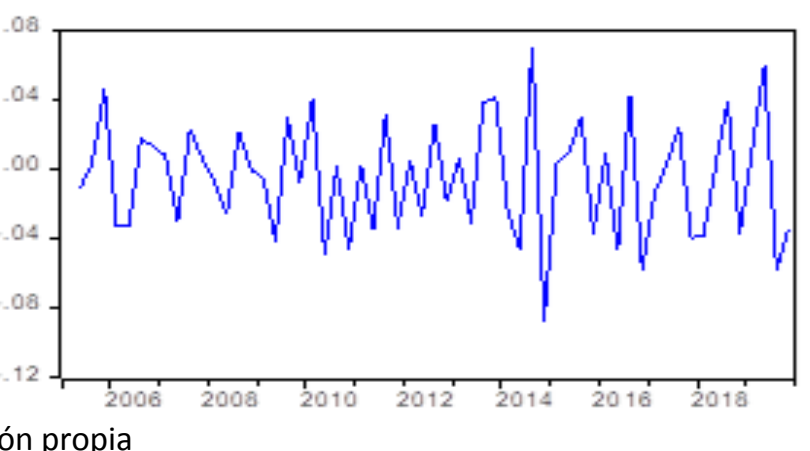

En la gráfica anterior con las variables de consumo de energía se aprecia que tienen un comportamiento estacional, el cual debe ser corroborado con la respectiva prueba.

Figura 7

Variables de tarifas de energía eléctrica del sector comercial, industrial, residencial y oficial, expresadas en logaritmos. 2005-2019.

Intecomercial

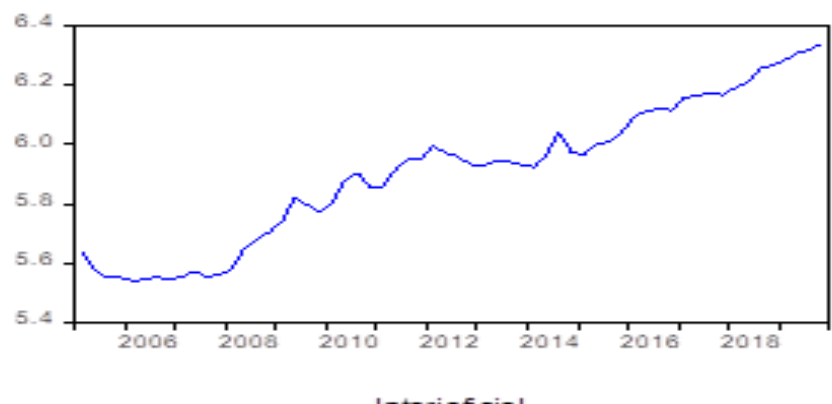

Interioficiel

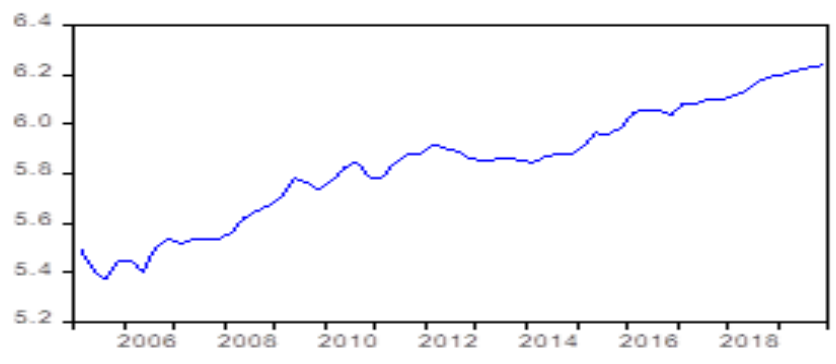

Intarindustrial

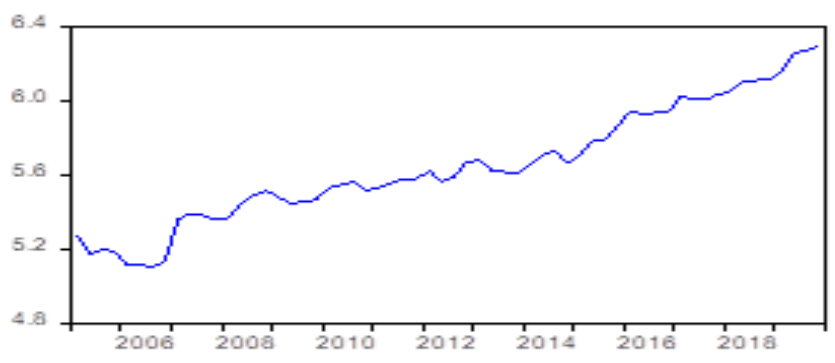

Inter ires idenciel

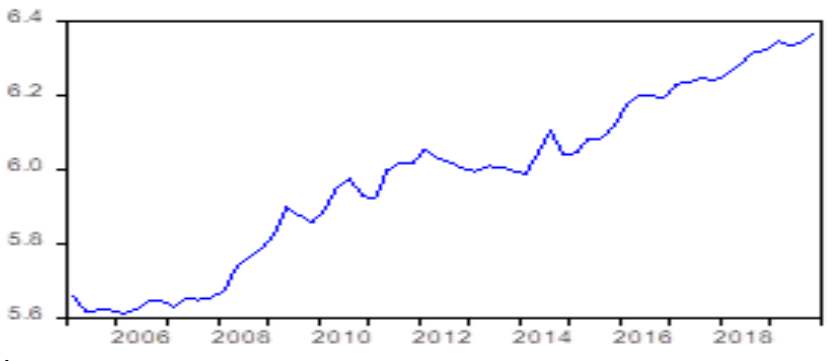

Fuente: Elaboración propia 
En la figura número seis, se puede apreciar que las variables no son estacionarias, por lo que se procede a la realización de la primera diferencia.

Figura 8

Variables de tarifas de energía eléctrica del sector comercial, industrial, residencial y oficial, expresadas en logaritmos y en primera diferencia. 2005-2019.

Differenced Intacomercial
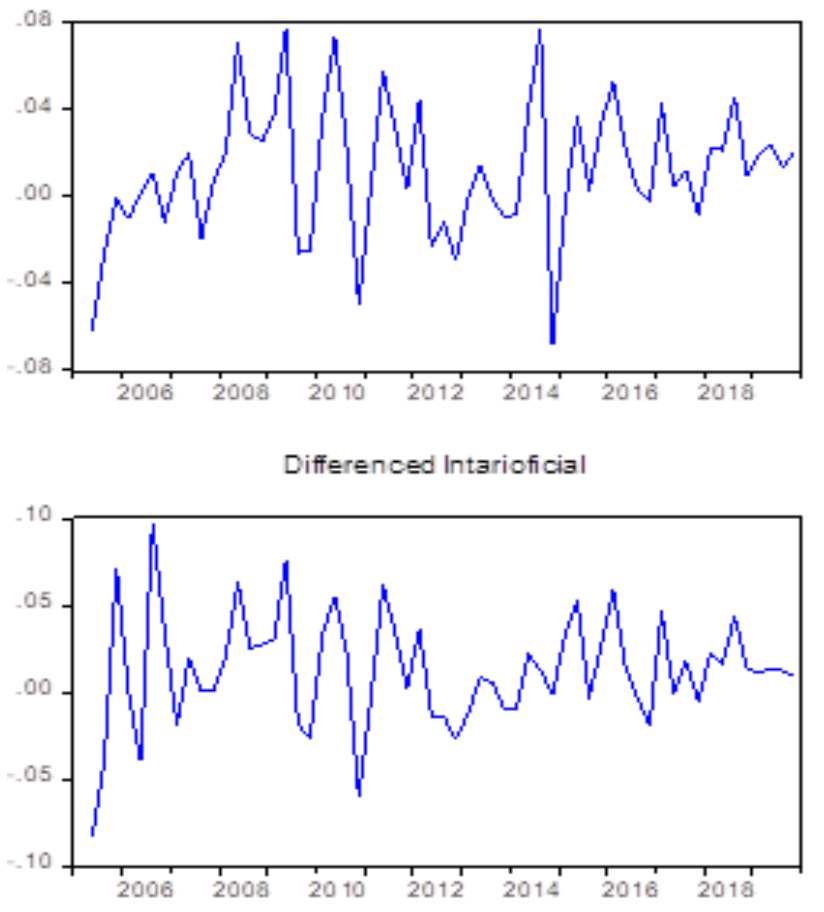

Differenced Intarindus trial
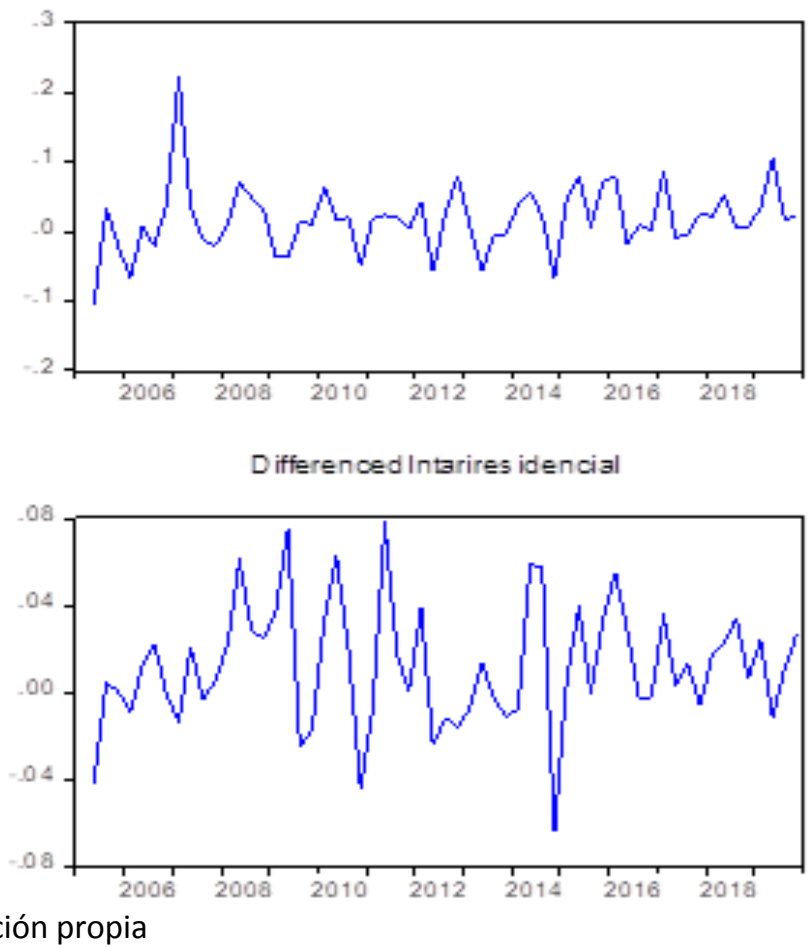

La figura número ocho permite observar que las variables de las tarifas al aplicarles la primera diferencia se comportan como variables estacionarias en media y en varianza.

En la figura número nueve, se grafican la Tasa de Ocupación para la ciudad de lbagué en forma logarítmica y en primera diferencia, la cual se comporta como variable estacionaria al aplicar la diferencia a la serie de tiempo. 


\section{Figura 9}

Tasa de Ocupación de la ciudad de lbagué, en logaritmos y en primera diferencia. 2005-2019.
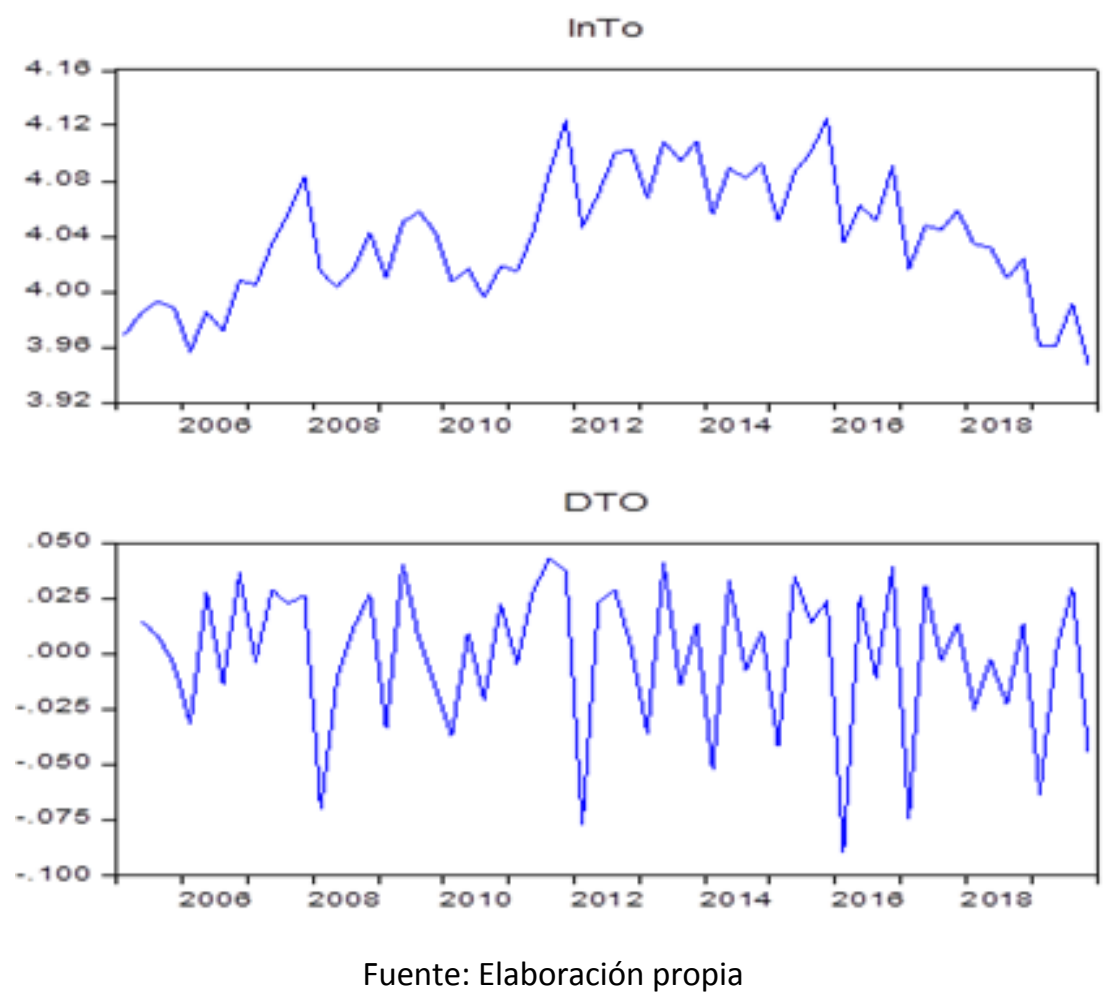

\subsection{Resultados obtenidos de la estimación por ARDL}

A, continuación se presentan los modelos obtenidos por ARDL de cada uno de los sectores que demandan energía, según como los clasifica la Superintendencia de Servicios Públicos Domiciliarios.

Modelo de la Demanda de Energía eléctrica en el sector comercio de la ciudad de lbagué.

DCONCOMER $=-0.089499747454 *$ DCONCOMER(-1) $+0.105718222295 *$ DTACOMERCIAL $\quad+$ 0.382939640741 *DTACOMERCIAL(-1) + 0.20273041661*DTO + 0.453274068531*DTO(-1) + 0.00249721847406

Modelo de la Demanda de Energía eléctrica en el sector residencial de la ciudad de Ibagué.

DCONRESIDEN = $-0.0538158945407 *$ DCONRESIDEN(-1) $-0.00110963684576 *$ DTARESIDENCIAL $\quad+$ 0.133090817281*DTARESIDENCIAL(-1) - 0.471459603031*DTARESIDENCIAL(-2) + 0.550455648075*DTO $4.43187001725 \mathrm{e}-05$

Modelo de la Demanda de Energía eléctrica en el sector industrial de la ciudad de lbagué.

DCONINDUSTRIAL $=-0.0578145671785 *$ DCONINDUSTRIAL(-1) $+0.0155042287585 *$ DTARINDUSTRIAL + $0.0263170589913 *$ DTO -0.00598425345289 
Modelo de la Demanda de Energía eléctrica en el sector oficial de la ciudad de lbagué.

DCONOFICIAL $=0.222696742985 *$ DCONOFICIAL(-1) $-0.281834791593 *$ DCONOFICIAL(-2) $\quad+$ $0.0711146573855 *$ DTAROFICIAL + 0.362603152975*DTO - 0.00638649196907

En el siguiente cuadro se recopila los resultados de los diferentes modelos estimados para la demanda de consumo de energía eléctrica en la ciudad de lbagué para los diferentes subsectores o clientes.

Cuadro 1

Resultados de las elasticidades de los modelos ARDL.

\begin{tabular}{lccc}
\hline Residencial & Corto plazo & Largo plazo & Coeficiente R \\
Precio & $-0,339469$ & $-0,322142$ & 0.286342 \\
TO & 0,550456 & 0,522345 & \\
& & & \\
Industrial & & & \\
Precio & 0.015504 & 0.014657 & \\
TO & 0.026317 & 0.024879 & 0.003916 \\
Comercial & & & \\
Precio & 0,488658 & 0.105718 & \\
TO & 0,20273 & 0.202730 & 0.307288 \\
& & & \\
Oficial & & & \\
Precio & 0.071115 & 0.067144 & \\
TO & 0.362603 & 0.342357 & 0.263103
\end{tabular}

Fuente: Elaboración propia.

\section{Conclusiones}

Con los modelos ARDL se estimaron las elasticidades de corto y largo plazo de la demanda de consumo de cada sector ante variaciones de los precios y de la tasa de ocupación de la ciudad de lbagué. En el cual las elasticidades precio de la demanda en el corto plazo arrojan el signo esperado para el sector residencial únicamente; caso contrario con la demanda precio para el resto de sectores, indicando que únicamente las familias son sensibles al incremento de las tarifas de la energía, pero lo hace de forma marginal. Los demás sectores no son sensibles al incremento de las tarifas de la energía, debido a que es un servicio que no se puede reemplazar de forma económicamente viable en los procesos de producción o prestación de servicios tanto en el sector privado, como en el público u oficial.

Referente a las estimaciones de la elasticidad de la tasa de ocupación en la demanda de energía en cada sector, los modelos ARDL predicen el signo esperado. Dando un mayor coeficiente para el sector residencial, los cuales aumentan el consumo a medida que logran tener trabajo dentro de la ciudad.

Los resultados de las elasticidades a corto plazo de los modelos ARDL permiten inferir una elasticidad precio de la demanda para los sectores de Comercial, Residencial e Industrial, e inelástica para el sector Oficial. Los 
resultados de las elasticidades para la variable Tasa de Ocupación arroja que es elástica para los sectores Industrial y Residencial, e inelástico para los sectores Comercial y Oficial evaluados en el corto plazo.

Las elasticidades estimadas por el modelo ARDL en el largo plazo, en lo referente a la elasticidad precio de la demanda para el sector residencial es significativa e inelástica, por lo que aumentos del precio de la energía significaría reducciones proporcionalmente menores al aumento del precio, dando igualmente significativa la elasticidad de largo plazo de la tasa de ocupación para el sector y con el signo positivo esperado. Al igual que la tasa de ocupación es significativa en el largo plazo para la demanda de energía eléctrica de los demás sectores analizados de la ciudad de Ibagué. Debido a esto debe ser prioridad para las autoridades de la ciudad brindar las condiciones para la generación de puestos de trabajo, puesto que dinamiza toda la economía, y como proxy del crecimiento económico.

Se recomienda seguir estudiando los diversos determinantes de la demanda de energía en la ciudad de lbagué, así como la evolución de los precios de la energía expresados en tarifas y sus efectos en los diversos sectores para realizar políticas de precios por parte de los agentes que intervienen en este mercado y que favorezca el crecimiento económico de la región, el empleo y el consumo de los diferentes sectores de la ciudad de lbagué.

Se recomienda en estudios futuros profundizar en la comprensión de la respuesta de las demandas de consumo de la energía eléctrica ante las variaciones en los precios y la dinámica económica y respondiendo a la pregunta

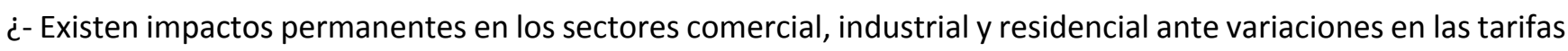
del precio de la energía eléctrica en la región y en el país?, así como el aporte del sector al crecimiento económico de la región y el país.

\section{Referencias bibliográficas}

AGOSTINI, Claudio; PLOTTIER, Cecilia; SAAVEDRA, Eduardo. La demanda residencial por energía eléctrica en Chile. ILADES-Universidad Alberto Hurtado. Chile. 2011.

Arias, E., \& Torres, C. (2004). Modelos VAR y VECM para el Pronóstico de Corto Plazo de las Importaciones de Costa Rica. Documento de Trabajo, (22).

AVILA FORERO, Raúl ESPINOSA ACUÑA, Oscar; VACA GONZALES, Paola. Elasticidades de demanda por electricidad e impactos macroeconómicos del precio de la energía eléctrica en Colombia. En: Revista de Métodos Cuantitativos para la Economía y la Empresa. Universidad Pablo de Olavide. Sevilla, España. 2013.

Ayala García, J. (2014). Crecimiento económico y empleo en Ibagué. BANCO DE LA REPÚBLICA-ECONOMÍA REGIONAL.

Baharumshah, A. Z., Mohd, S. H., \& Masih, A. M. M. (2009). The stability of money demand in China: Evidence from the ARDL model. Economic systems, 33(3), 231-244.

BARRETO NIETO, Carlos Alberto; CAMPO ROBLEDO, Jacobo. Relación a largo plazo entre consumo de energía y PIB en América Latina: Una evaluación empírica con datos panel. En: Ecos de Economía. ISSN 1657-4206 I Año 16 I No. 35 I julio-diciembre 2012 I pp. 73-89 I Medellín-Colombia.

BARRIENTOS, Andrés Felipe; OLAYA, Javier; GONZÁLEZ, Víctor Manuel. Un modelo spline para el pronóstico de la demanda de energía eléctrica. En: Revista Colombiana de Estadística. Volumen 30 No. 2. pp. 187 a 202. Cali. 2007 
BENAVENTE, José Miguel; GALETOVIC, Alexander; SANHUEZA; SERRA Pablo. Estimando la demanda residencial por electricidad en Chile: el consumo es sensible al precio. En: Cuadernos de Economía, vol. 42 (mayo), pp. 31-61. Chile. 2005

BLACONÁ, María Teresa; ABRIL, Juan Carlos (2000). Modelo Estructural de Espacio de Estado para la demanda diaria promedio de energía eléctrica en la República Argentina. ITAE, Argentina.

BOTERO, Jesús Alonso; VÉLEZ, Carlos Eduardo; YAÑEZ, Sergio. La demanda residencial de electricidad: un caso colombiano. 1970-1983. En Lecturas de Economía №34. 1991. Medellín.

BOTERO, Jesús; CASTAÑO, Elkin; VÉLEZ, Carlos Eduardo. Modelo económico de demanda de energía eléctrica en la industria colombiana. Centro de Investigaciones Económicas -CIE. Universidad de Antioquia. En: Lecturas de Economía. No. 32-33. Medellín. 1990

CARDONA ACEVEDO, Marleny; OCAMPO CASTAÑO, Dora María. Demanda de energía eléctrica del sector residencial en el área metropolitana (Medellín). Trabajo de grado presentado como requisito parcial para optar por el título de economista. Universidad de Antioquia. 1989. Medellín.

Cavaliere (2003) "Vector autoregression models", Study Centre Gerzensee CBC Course.

FRANCO, Carlos Jaime; VELASQUEZ, Juan David; OLAYA, Yris. Caracterización de la demanda mensual de electricidad en Colombia usando un modelo de componentes no observables. En: Cuadernos de Administración, UNAL. Bogotá. 2008.

GALINDO VARGAS,Andrea Paola. La relación entre el consumo de electricidad y el crecimiento económico empleando un modelo trivariado para Chile. Tesis de maestría en ciencias económicas. Universidad Nacional de Colombia 2013.

Gujarati, D. N., \& Porter, D. C. (2011). Econometria Básica-5. AMGH Editora.

MEDINA, Eva; VICÉNS José. Factores determinantes de la demanda eléctrica de los hogares en España: Una aproximación mediante regresión. En: Estudios de Economía Aplicada. VOL. 29 - 2 2011. Pág. 515 - 538.

MEDINA, Santiago; GARCÍA, Josefina. Predicción de demanda de energía en Colombia mediante un sistema de inferencia difuso neuronal. En: Energética, núm. 33, Universidad Nacional de Colombia. Medellín, Colombia. 2005

Misas, M., \& Hugo, O. (1997). Cointegración, exogeneidad y crítica de Lucas: funciones de demanda de dinero en Colombia: un ejercicio más. Borradores Semanales de Economía.

MURILLO, Joaquín; TREJOS, Álvaro; CARVAJAL, Patricia. Estudio del pronóstico de la demanda de energía eléctrica, utilizando modelos de series de tiempo. En: Revista Scientia et Technica Año IX, No 23, UTP. Pereira. 2003.

Novales, A. (2011). Modelos vectoriales autoregresivos (VAR). Universidad Complutense.

Ortiz, A. M., Afanador, E., Zapata, J. G., Núñez, J., Ramírez, R., Yepes, T., \& Garzón, J. C. (2013). Análisis de la situación energética de Bogotá y Cundinamarca. FEDESARROLLO-EMPRESA DE ENERGÍA DE BOGOTÁ.

Pahlavani, M., Wilson, E., \& Worthington, A. C. (2005). Trade-GDP nexus in Iran: An application of the autoregressive distributed lag (ARDL) model.

TABARES, Héctor; HERNÁNDEZ, Jesús. Mapeo curvas típicas demanda de energía eléctrica del sector residencial, comercial e industrial de la ciudad de Medellín, usando redes neuronales artificiales y 
algoritmos de interpolación. En:Revista de la Facultad de Ingeniaría de la Universidad de Antioquia N. 46 pp. 110-118. Diciembre, 2008

TAYLOR, Lester D.TheDemandforelectricity: a survey. En: Bell Journal of Economics. 1975. Estados Unidos.

UNIDAD DE PLANEACCIÓN MINERO ENERGÉTICA. Proyección de Demanda de Energía Eléctrica en Colombia.Informe de Marzo de 2013

VELÁSQUEZ, Juan David; FRANCO, Carlos ; GARCÍA, Hernán. Un modelo no lineal para la predicción de la demanda mensual de electricidad en Colombia. Universidad Nacional de Colombia sede Medellín.

Medellín. 2009

ZAPATA, Carlos; LÓPEZ, Julián; RENGIFO, Erickson. Efecto del modelamiento de la demanda en estudios de confiabilidad de largo plazo de sistemas eléctricos. En: Revista Scientia et Technica Año XII, No 32, UTP.

\section{Anexos}

\section{Anexo 1}

Tabla de resultados de la prueba de raíz unitaria para todas las variables

\begin{tabular}{|c|c|c|c|c|c|c|c|c|c|}
\hline \multirow{2}{*}{ 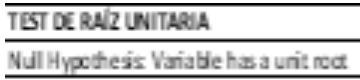 } & \multicolumn{9}{|c|}{ VARIABLES } \\
\hline & ICCOMER & LCNOUST & LCOFC & LQRES & LPCOMER & LPNDUST & LPOFCIM & pacs & LTOI \\
\hline \multicolumn{10}{|l|}{ LOFintertepto tendercia } \\
\hline Astatific & -4456.174 & -1853045 & -3.336 .200 & -6077.866 & -2103862 & -1.171 .426 & -161130 & -168.197 & -3.990 .803 \\
\hline Ard." & 00044 & 06039 & 0.0720 & 00000 & 0.5150 & 0.0000 & 00398 & 0.7429 & 0.0270 \\
\hline \multicolumn{10}{|l|}{ LDF-inter cepto } \\
\hline Atatisic & -4034665 & -181867 & -3.982 .693 & -6072450 & -1340791 & -1.140 .986 & -2249395 & -0.860043 & -0.680222 \\
\hline Arda." & 00027 & 0.366 & 0.0037 & 0,000 & 06033 & 0.0000 & 0.1924 & 0.3390 & 0.8409 \\
\hline \multicolumn{10}{|l|}{ AOF-niguna } \\
\hline Statisic & 1014898 & 0586328 & -0.352530 & 0.099480 & -102615 & -0386772 & -0860043 & -0.90262 & 1064333 \\
\hline Arda." & 09159 & 08398 & 0.5530 & 07093 & 0.2415 & 0.5292 & 0.3380 & 03176 & 0.9224 \\
\hline Null Hyxtesis Variable hæs unit nod. & DICCOMLR & DCINDUST & DucofC & acres & DPCOMER & opingust & DQPOFCIN & Dlpacs & DATOI \\
\hline \multicolumn{10}{|l|}{ LOFintercepto tendencia } \\
\hline Mtribjic & -6415248 & -7.996424 & -7.934 .634 & -7.966 .307 & -9037.320 & -4.766 .555 & -1441204 & -5.754 .167 & -2.423 .236 \\
\hline Arch." & 0,000 & 0000 & 0.0000 & 0000 & 0000 & 0.0021 & 0058 & 0.0001 & 0.300 \\
\hline \multicolumn{10}{|l|}{ LOF-intercepto } \\
\hline Atstistic & 6323196 & -7.69380 & -8.020 .806 & -8029876 & -2133589 & -5.170 .54 & -3592903 & -5817947 & -2.499 .138 \\
\hline Prch." & 0000 & 0000 & 0.0000 & $000 \infty$ & 0000 & 0.0001 & 00097 & 0.0000 & 0.1274 \\
\hline \multicolumn{10}{|l|}{ LDF-nigura } \\
\hline Atstistic & -6227.434 & -7.627 .847 & 8.115 .913 & -8115913 & -2057.561 & -5.399 .330 & -156587 & -5.764672 & -2.129 .009 \\
\hline Ard." & 0000 & 0000 & 0.0000 & 0000 & 0000 & 0.0000 & 00007 & 0,0000 & 0.0000 \\
\hline
\end{tabular}

Esta obra está bajo una Licencia Creative Commons Attribución-NoCommercial 4.0 International

(cc) EY-NO 